\title{
Lorentz Factor Evolution Patterns within Relativistic Jets of GRBs and AGNs
}

\author{
Hai-Ming Zhang, Da-Bin Lin*, Ting-Ting Lin, Bao-Rong Liu, \\ Xiao-Li Huang, Shu-Qing Zhong, Rui-Jing Lu and En-Wei Liang* \\ Guangxi Key Laboratory for Relativistic Astrophysics, School of Physical Science \& \\ Technology, Guangxi University, Nanning 530004, China \\ GXU-NAOC Center for Astrophysics and Space Sciences, Guangxi University, Nanning \\ 530004 , China \\ email: lindabin@gxu.edu.cn; lew@gxu.edu.cn
}

\begin{abstract}
The Lorentz factor $(\Gamma)$ is an important parameter related to the relativistic jet physics. We study the evolution patterns of $\Gamma$ within gamma-ray burst (GRB) and active galactic nuclear jets for individual GRB 090168, GRB 140508A, and 3C 454.3. By estimating the $\Gamma$ values for well-separated pulses in GRBs 090618 and 140508A with an empirical relation derived from typical GRBs, we find that the $\Gamma$ evolution pattern in the two GRBs are different. The increasing-to-coasting evolution pattern of $\Gamma$ in GRB 090618 likely indicates that the GRB fireball is still being accelerated in the prompt phase. The clear decrease evolution pattern of $\Gamma$ in GRB 140508A suggests the deceleration of the fireball components. By deriving the $\Gamma$ value through fitting their spectral energy distribution in different flares of 3C 454.3, a pattern of $\Gamma$-tracking- $\gamma$-ray flux is clearly found, likely indicating that the observed gamma-ray flares are being due to the Doppler boosting effect to the jet emission.
\end{abstract}

Keywords. gamma rays: bursts (individual: GRBs 090168 \& 140508A), active galactic nuclei: individual (3C 454.3), jets and outflows.

\section{Introduction}

Gamma-ray bursts (GRBs) are the most explosive events in the universe. It is believed that the prompt emission of GRBs is produced in an ultra-relativistic jet. A relativistic jet is also widely found in active galactic nuclei (AGNs). For a relativistic jet, its Lorentz factor $\Gamma$ is an important parameter to understand its physics, such as the jet composition and acceleration process, etc. In this work, we focus on the Lorentz factor of GRB pulses or gamma-ray flares of AGN jets.

Several methods have been proposed to infer $\Gamma$ values of GRBs. The most popular method is by taking the peak time of the early afterglow light curve as the deceleration time of the external forward shock (e.g., Sari \& Piran 1999). One may also estimate the $\Gamma$ value with the high-energy cutoff observed in the prompt gamma-ray spectrum based on the "compactness problem" constraint (Fenimore et al. 1993; Piran 1999) or with the blackbody emission component (Pe'er et al. 2007). Interestingly, a tight relation of $\Gamma_{0}$, $L_{p \text {,iso }}$ and $E_{p, z}$ is found with a sample of GRBs whose early optical afterglow onset peaks are clearly detected (Liang et al. 2015). To estimate the $\Gamma$ value of an AGN jet, one needs to measure its Doppler boosting factor $(\delta)$ and the viewing angle $\theta_{v}$ to the jet axis. The $\delta$ value may be estimated with very long baseline interferometry observations (e.g., Ghisellini et al. 1993), the variability brightness temperatures (Lähteenmäki \& Valtaoja 1999; Hovatta et al. 2009), or the variable timescale of sources (Jorstad et al. 2005). Theoretically, the Doppler factors may also be constrained by modeling the observed broadband spectral energy distributions (e.g., Zhang et al. 2012). For blazars, it is generally believed 


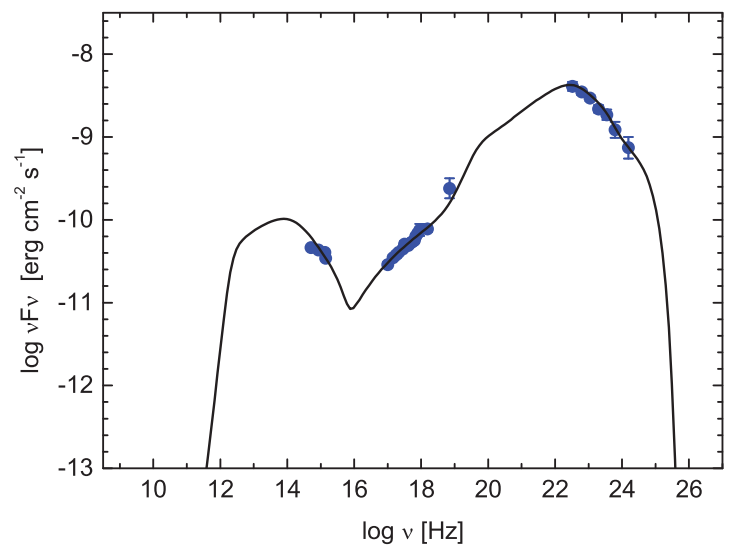

Figure 1. Spectral fitting of observations (points) of 3C 454.3 at MJD 55168.0. The solid line is our spectral fitting result.

that $\theta_{v}$ is very small and $\delta=\Gamma$ is usually adopted in theoretical modeling. This paper studies the evolution patterns of $\Gamma$ within individual GRBs and blazars.

\section{Data}

We select GRBs $090618(z=0.45)$ and 140508A $(z=1.03)$ for case study. They have well-separated gamma-ray pulses with different peak fluxes. Their Fermi/GBM light curves are showed in Figure 2. We use the empirical $L_{p}-E_{p, z}-\Gamma_{0}$ relation relation (Liang et al. 2015), i.e., $\log \Gamma_{0}=3.33+0.46 \log L_{p, \text { iso }, 52}-0.43 \log E_{p, z}$, to estimate the $\Gamma$ values for these pulses. We extract the spectra of these pulses around the peak times $\left(\left[t_{p}-0.5 \mathrm{~s}, t_{p}+0.5 \mathrm{~s}\right]\right)$ and fit them with the Band function to derive their $E_{\mathrm{p}, z}=E_{p}(1+z)$ and $L_{p, \text { iso, } 52}$.

3C $454.3(\mathrm{z}=0.859$, also named PKS $2251+158)$ is a blazar. It is the brightest gammaray source in the gamma-ray sky. Fermi Large Area Telescope has detected many bright flares for this source since it was launched in 2008 (Abdo et al. 2009). We extract its 8 years' LAT lightcurve and select some bright flares occurred in the period of [MJD55100, MJD55240] and [MJD55480, MJD55600] for our analysis, as shown in Figure 3 , where only photons with energy $100 \mathrm{MeV}<E<200 \mathrm{GeV}$ are considered. The $\Gamma$ values of these flares are estimated based on the broad band SED (spectral energy distribution) fitting with the simple one-zone leptonic models (Bonnoli et al. 2011, Pacciani et al. 2010, Vercellone et al. 2011, Cerruti et al. 2013) as shown in Figure 1 for an example.

\section{Results}

Our results are shown in Figures 2 and 3. Different evolution features are found in the two GRBs. An increasing-to-coasting $\Gamma$ evolution pattern is found in GRB 090618, likely indicating that its fireball is still being accelerated in the initial prompt gamma-ray phase, then kept at almost constant speed. A clear decrease evolution pattern of $\Gamma$ in GRB $140508 \mathrm{~A}$ is found. This likely suggests the deceleration of the fireball components for different pulses. These results may imply that there is no universal evolution pattern among GRBs. For 3C 454.3, a pattern of $\Gamma$-tracking- $\gamma$-ray flux is clearly found in different flares. 

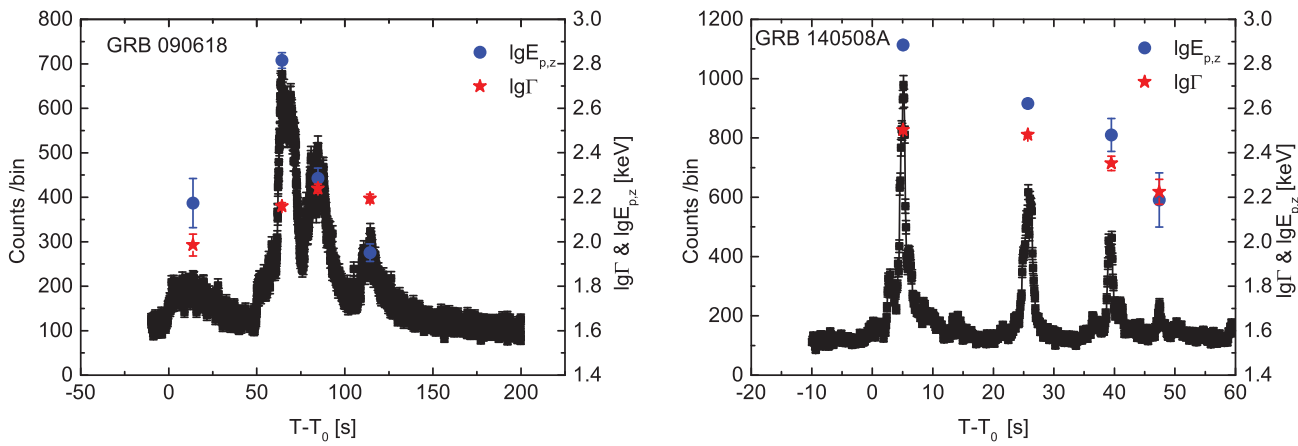

Figure 2. Evolution of photon counts (squares), $\Gamma$ (stars), and $E_{p, z}$ (dots) in GRB 090618 (left panel) and GRB 140508A (right panel). $T_{0}$ is the trigger time of the burst.
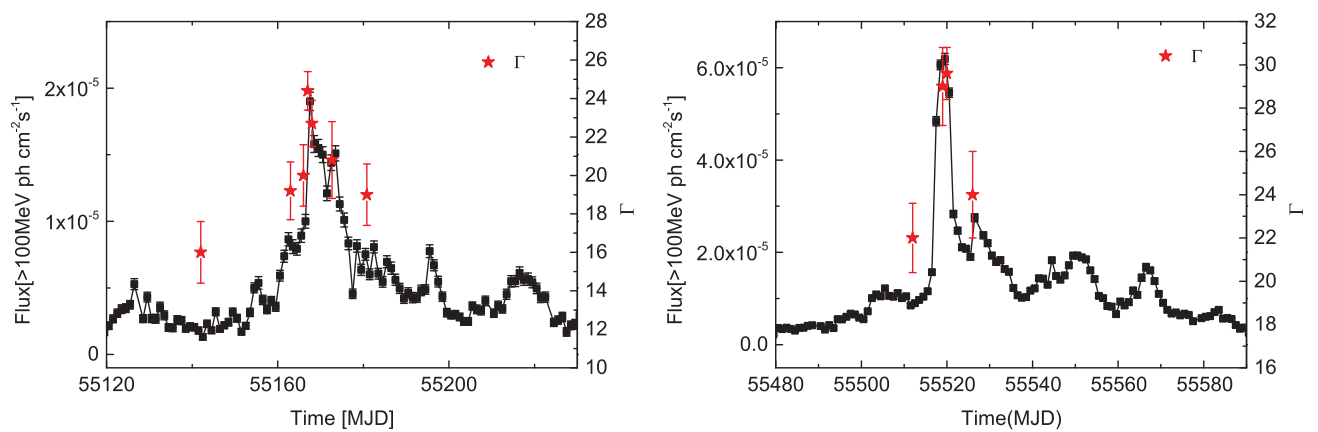

Figure 3. Evolution of flux and $\Gamma$ (stars) in 3C 454.3 for observations in December 2009 (left panel) and November 2010 (right panel). The error bars of flux are statistical only.

\section{Discussion \& Conclusion}

We have studied the $\Gamma$ evolution within GRBs 090618 and $140508 \mathrm{~A}$ as well as within 3C 454.3. It is found that the $\Gamma$ evolution patterns in the two GRBs are different. This may suggest that there is no universal evolution pattern among GRBs. The widely accepted GRB model is the fireball model. Within the framework of this model, the prompt gamma-ray emission spectrum is expected to be the superposition of a quasi-thermal photosphere emission component and a non-thermal component in the optically thin internal shock region. It was proposed that the spectrum of the first pulse in GRB 090618 is superimposed by such a thermal component (Campana et al. 2006). This likely suggests that the emission of this pulse may come from the region around the photospheric radius of the fireball. The pure non-thermal emission in the next three pulses may be attributed to the emission from pure internal shock region. Therefore, the increase of $\Gamma$ would suggest that the fireball is still accelerated near the photospheric radius. The $\Gamma$ value keeps almost constant in the next three pulse which suggests that the fireball is within the coasting state. For GRB 140508A, the clear decrease of $\Gamma$ may be due to the decay of the GRB central engine activities since a random collision process for fireball shells has difficulties in explaining this feature. For 3C 454.3, a pattern of $\Gamma$-tracking- $\gamma$-ray flux is clearly found. If this is a universal feature, it may suggest that the observed gamma-ray flares would be due to the Doppler boosting effect to the emission from ejecta within a jet powered by the central black hole. Gamma-ray flares of AGNs thus may be a signal of the central engine activity. 


\section{Acknowledgements}

We acknowledge the use of the public data from the Fermi data archive. This work is supported by the National Basic Research Program of China (973 Program, grant No. 2014CB845800), the National Natural Science Foundation of China (Grant No. 11533003, 11403005, 11463001, 11363002), the Strategic Priority Research Program The Emergence of Cosmological Structures of the Chinese Academy of Sciences (grant XDB09000000), the Guangxi Science Foundation (2016GXNSFDA380027, 2014GXNSFAA118011, 2014GXNSFBA118004).

\section{References}

Abdo, A. A., Ackermann, M., Ajello, M., et al. 2009, ApJ, 699, 817

Bonnoli, G., Ghisellini, G., Foschini, L., et al. 2011, MNRAS, 410, 368

Campana, S., Mangano, V., Blustin, A. J., et al. 2006, Nature, 442, 1008

Cerruti, M., Dermer, C. D., Lott, B., Boisson, C., \& Zech, A. 2013, ApJL, 771, L4

Fenimore, E. E., Epstein, R. I., \& Ho, C. 1993, A\&AS, 97, 59

Ghisellini, G., Padovani, P., Celotti, A., \& Maraschi, L. 1993, ApJ, 407, 65

Hovatta, T., Valtaoja, E., Tornikoski, M., \& Lähteenmäki, A. 2009, A\&GA, 494, 527

Jorstad, S. G., Marscher, A. P., Lister, M. L., et al. 2005, AJ, 130, 1418

Lähteenmäki, A. \& Valtaoja, E. 1999, ApJ, 521, 493

Liang, E.-W., Lin, T.-T., Lü, J., et al. 2015, ApJ, 813, 116

Pacciani, L., Vittorini, V., Tavani, M., et al. 2010, ApJL, 716, L170

Pe'er, A., Ryde, F., Wijers, R. A. M. J., et al. 2007, ApJ, 664, L1

Piran, T. 1999, Phys. Rep., 314, 575

Sari, R. \& Piran, T. 1999, ApJ, 520, 641

Vercellone, S., Striani, E., Vittorini, V., et al. 2011, ApJL, 736, L38

Zhang, J., Liang, E.-W., Zhang, S.-N., \& Bai, J. M. 2012, ApJ, 752, 157 